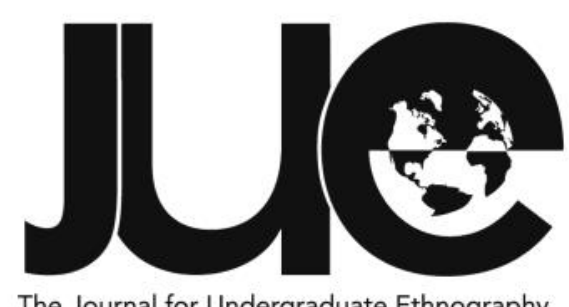

The Journal for Undergraduate Ethnography

\title{
Vitiligo: Challenging Cultural Assumptions and Shaping Identity Hamna Khuld
} Montclair State University, Khuldh@alumni.montclair.edu

\section{ABSTRACT}

Vitiligo is a skin condition where pigmentation stops developing, leaving people with white spots on their bodies. Vitiligo is likely caused by gene mutation and is hereditary, but it can happen to anyone. From a medical standpoint, it is a physically harmless condition but it has vast socio-cultural impact. This study was conducted at the Annual World Vitiligo Conference in Detroit, Michigan and on the internet (Instagram and Facebook), through participant-observation at the event, textual analysis of blog posts, and interviews online and in-person, respectively. Through these methods, three discourses emerged: 1) Feeling outcast, 2) Vitiligo as beautiful, and 3) Solidarity. I documented the way cultural assumptions about conditions and disabilities shape the identity of those who have it. These interviews suggest that vitiligo is as much a cultural condition as it is a medical condition. Although more research is needed, people living with vitiligo stated that greater representation of individuals with the condition is needed in the media and pop culture to enlighten the public about vitiligo and improve the day to day interactions of individuals with the condition.

Keywords: vitiligo, race, gender, normalization, animalization, stigma, United States 


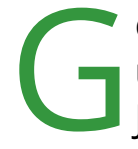
etting on the bus after a busy day of university classes, a young lady named Jasmine looked around to see if there was an available space to sit. She approached a follow passenger and asked, "Excuse me, is this seat taken?" pointing at the seat next to the man. The passenger looked at her for a while, and then pushed his belongings next to the window to his side and scooted away from her. Jasmine remained quiet and took her seat, trying hard not to show this interaction was impacting her, even when the man continued to glance at her. She knew why her fellow passenger acted the way he did: it was due to the white patches adorning her mocha skin. The man was looking at her vitiligo and contemplating if it was contagious.

Jasmine's experience was not unique. Throughout my research, participants have shared similar instances of daily life with vitiligo. They shared stories of overcoming stigma they are culturally subjected to, forming communities through various online and faceto-face platforms, and eventually learning to accept, identify with, and embrace their condition.

\section{Medical Treatment}

Though vitiligo has no cure, clinicians offer a handful of medical treatments consisting of Steroid cream, ultraviolet laser therapy (UVB), and makeup, which is classified as a solution. Despite the wealth of products for sale to treat the condition, very little, if any, research suggests that these treatments are effective in minimizing the presence of spots, let alone prevent future de-pigmentation. Furthermore, some individuals have utilized ultraviolet therapy (UVB), but many fear it might harm their skin due to the lack of melanin and inevitably stop the treatment. Even if these medical treatments prove effective, treatment is pricey and most individuals cannot afford it or their insurance will not cover the cost for it.

\section{Background: The Cultural Life of Vitiligo}

Even though vitiligo is a medical condition and can be examined as such, this paper focuses on the sociocultural aspects of the condition as a disability, and examines the social stigma, ostracization, animalization, and other barriers faced by individuals with vitiligo.

In the United States and across other cultures, the implications of changes to skin pigmentation have repercussions for both personal identity and group inclusion vis-à-vis race and ethnicity. Furthermore, because the skin is a highly visible symbol of identity and well-being, changes to it can raise concerns about the unknown, including fears of witchcraft and contagion. Many individuals fall victim to harsh comments, antagonism, and ostracization, which leads to the development of anxiety, depression, and low self-esteem (Parsad, Dogra, and Kanwar 2003, 54).

Vitiligo is a unique condition, in that the outward skin condition can disrupt racial identity through loss of pigmentation. While it may seem anachronistic in 2018 that people would be ostracized, shunned, or evicted from their communities, churches, or homes as a result of skin depigmentation, the stigma surrounding the condition in the United States persists. According to Porter and Buef (1991), people of color who develop vitiligo feel they are losing their racial identity; thus, they are faced with exclusion by communities and family because they are considered an "other." As presented by Porter and Buef (1991), black individuals with vitiligo are more severely affected by stigma than their white counterparts and prone to lose their "master status" such as race, age, and gender (194). Instead, disability becomes their master status. Vitiligo affects black individuals more negatively than white individuals because the depigmentation is highly visible on darker skin and is much more difficult to hide through available cosmetics. Goffman (1963) pioneered the idea of stigma as a "spoiled identity," which has been used above to explain how lightning 
skin can be understood as a threat to racial identity, but also how people themselves internalize cultural norms about themselves (140). This is sometimes referred to as “internalized ableism" (Campbell 2008, 153). The importance of appearance may be less critical for black men compared to black women because of gender roles and norms. My research suggests that high levels of perceived stigma could be related to distress, the importance placed on a cure, labeling vitiligo as a disability, and the visibility of depigmentation. In addition, for both youths and for women, disfigured appearance may be socially stigmatized more heavily because appearance is a central feature of role expectations for these statuses.

This is evident in the United States, where individuals of certain races and ethnic groups do not face the same amount of stigmatization because of their complexion. For example, darker skin results in more stigma while lighter skin receives less stigma. Individuals with darker skin are more inclined to be questioned and stared at as a result of their vitiligo. Thereby illustrating that vitiligo is a cultural construction that causes individuals to be judged or stigmatized.

\section{Methods}

Presented below are first-person narratives of living with vitiligo. As a participant at the 2017 Annual World Vitiligo Conference in Detroit, Michigan, I utilized participation-observation (attended workshops, listened to speakers, and gathered information from tablings) and I conducted informal interviews at the site and via online platforms (Instagram and Facebook). Through these methods, I discovered recurring issues in in-person interviews and online platforms that consisted of stigma and ostracization, where individuals are thought of as "freaks/different/others." I listened to presenters speak about their experiences with vitiligo and the hardships they faced in accepting the condition and bringing attention to it. I observed the language being used in defining vitiligo and the audience's response to the specific details that were shared by the presenters. During the 15 to 30 minute intermissions I approached the attendees and conveyed to them that I was interested in interviewing them. I provided the potential participants with my contact information and asked them to reach out to me if they were interested in participating.

The conference was attended by a variety of people. Not everyone had vitiligo; there were family members and friends there, just like myself. I do not have vitiligo but a few members of my nuclear family do. I was not excluded from the event. Instead, individuals were very open about sharing their experiences when I asked them if they would like to participate in my research. I would like to note that all participants' names are pseudonyms unless otherwise requested (e.g., they are known on Instagram and want to be recognized, so they preferred their real name be used.)

In the following weeks, seven participants ranging in age from their early 20 s to late 40 s from diverse backgrounds reached out to me to set up time for an interview. They were eager to speak about their experiences with vitiligo. I wanted to interview people from various backgrounds because they had different experiences, impacted by the color of their skin, age, and gender. Participants were incredibly forthcoming in in-person interviews; they were already at least somewhat comfortable in their own skin, but they did not seem to hold back in sharing personal details, nor did I seem to make them uncomfortable by talking about what might otherwise be personal issues.

Additionally, I checked support sites, blogs, and social media sites (Facebook and Instagram) to see how individuals with vitiligo connected online over their condition, especially since a few participants alluded to having started hashtags such as; \#VitiligoPride, \#DopplerBodies, and \#SpottedBeauty. Through these hashtags, pictures, and blogs I saw various comments and posts illustrating that many individuals accepted their condition and formed a community where people shared their journeys and supported and complemented each other's vitiligo.

Furthermore, I wanted to comment on the differences between in-person interviews and informants from online platforms (Facebook and Instagram). These methods addressed 
issues of stigma and exclusion, acceptance of the condition, and solidarity. These online platforms, which are highly visual, exhibit a wider network of people with the condition, and not only create visibility and solidarity for the community but also promote discussion. The platforms function like group interviews, where individuals share their experiences through various formats, such as pictures of their vitiligo on parts of the body or of tattoos commemorating the condition, or stories and discussions of stigma, ostracization, animalization, treatments, and acceptance. I noticed no difference in the quality or amount of data I received from the different sources- in -person interviews revealed just as much sensitive information as online chats. My presence did not seem to make people uncomfortable or reticent to reveal sensitive information, which I initially expected might be easier to gather online where people have more privacy.

\section{Theoretical Framework}

My participants' experiences have informed the theoretical approach I take to vitiligo. Specifically, the notions of stigma, normalcy, and disability illuminate the skin condition as cultural, not just a medical issue. Many lay people conceptualize "stigma" as a harmless consequence, a relatively insignificant effect they need to put up with or even as a normal part of life in American culture. That could not be farther from the truth. Stigma, according to the clinical literature by Koo and Lee (2003, 3589), has been shown to lead to low self-esteem, depression, anxiety, and even an increase in psychiatric morbidity. Koo and Lee (2003) mentioned a study carried out in the U.S. with patients of vitiligo. The study compared how people with vitiligo deal with their skin condition compared to patients with psoriasis and other pigment skin disorders (hypo or hypermelanosis) in social stigma. It was found that participants with vitiligo had lower selfesteem than those without skin diseases, but maintained the same psychosocial effects as others with skin conditions. However, patients with vitiligo exhibited better adjustment to their condition and experienced less social discrimination than their more prominent skin condition counterparts (358). In Koo and Lee's
(2003) study, they find that individuals with skin conditions such as vitiligo express feelings of stress, embarrassment, or self-consciousness when in casual contact with strangers, which leads to low self-esteem and difficulty maintaining relationships. The psychological impact can be of paramount importance in deeply pigmented races, in whom the contrast between normal and affected skin is marked, and the disease can carry a severe social stigma (358). Goffman (1963) explained there are different types of stigma that categorize "abominations" of the body, which include various physical deformities; this is the type of stigma endured by individuals with vitiligo, where the body is loathed by society (as cited in Davis 2006, 132). Goffman (1963) further reveals that "disparaging reactions from other services to invalidate the disabled person as less than "normal" if not less than "human" beings" (as cited in Berger 2013, 9). This type of stigma is understood as a key characteristic attached to a person that "reduces [the individual] in our minds from a whole and usual person to a tainted discounted one" (Goffman $1963,35)$. Stigma prompts the public to see the individual with the disability or condition as a faulted person, identified only by his or her impairment, considering it the individual's "master status" (Goffman 1963, 35).

The theory of normalcy also pertains to people with disabilities and the discrimination and the disadvantages they face. Davis (2006) redefined the concept of the "normal" in relation to the general population, coming up with three variations. First, the application of the idea of a norm applied to the human body creates the idea of deviance or a "deviant" body. Second, the idea of norms pushes the normal variation of the body through a stricter template guiding the way the body "should" be. Finally, the revision of the "normal curve of distribution" into quartiles can be ranked in order, to create a new kind of ideal (Davis 2006, 8). The normal curve of distribution was developed to introduce a precise way of speaking of the typical, or the average, or the mean, thus providing a descriptive and objective aspect. According to Parens (2006), "The statistical norm provides a representation of what is - as in the typical, or the average, or the mean...[the] average seems to be distant 
from the ideal...[therefore] the average can become an ideal" (94). The normal curve of distribution epitomizes a statistical understanding of "normal," where the ideal body shifts based on the presumed descriptive norm of normal, a prescriptive force (as cited in Parens 2006, 94). Because of the normal curve of distribution people with disabilities and conditions are outcast by the larger society of fear of being different or contagious. Goffman (1963) illustrated that normalcy is a standpoint that does not obtrude but rather allows for the recognition of whom or what is stigmatized (as cited in Titchkosky \& Michalko 2009, 43).

Normalcy also contributed to the establishment of the "Ugly Law," which stipulated that it was illegal to appear in public if one's physical appearance offends others in the general public. This law was enacted in various cities across the United States, resulting in barring or preventing people with disabilities and conditions from appearing in public. According to Berger (2013), the city of Chicago passed an ordinance instituting the Ugly Law in 1881 and only repealed it in 1973. The government officials wrote in regard to the law:

Any person who is diseased, maimed, mutilated, or in any way deformed, so as to be an unsightly or disgusting object or an improper person to be allowed in or on the streets, highways, thoroughfares, or public places in this city, shall not therein or thereon expose himself to public view under the penalty of a fine of $\$ 1$ (about \$20 today) for each offense (7).

This effectively made people with disabilities outcasts because they did not fit into the norm. Even though this law is no longer enacted, for many individuals with disabilities and conditions the effects are still felt. Lucy Grealy, whose face was disfigured due to surgery for facial bone cancer, recalled with "great pain the cruel stares and laughter she encountered and state[ed] "I was my face, I was ugliness"(Beger 2013 , 8). Grealy's words illustrate how people with disabilities often experience prejudice and discrimination leading to marginalization and disadvantages comparable to what is experienced by people of color and other minority populations. This sadly remains true, and bodies that are different from "a society's conception of a 'normal' or acceptable body," even when it causes "little or no functional or physical difficulty for the person who has them, constitute major social disabilities" (Berger 2013 , 8). This discrimination faced by individuals is reflective of the normal distribution curve, where individuals with abnormalities, including skin conditions like vitiligo, are outcast and othered by the general public because they do not fit into the ideal acceptable body.

Compared to normalcy, animalization is used to separate, dehumanize, and objectify people through animal comparisons and slurs. Taylor (2017) explains that animalization faced by people with disabilities is not only insulting - it poses the risk of the loss of their personhood since the slurs remove them from "humanity" and places them in the category of animals (105). This is further illustrated by Clare (2017), who showed how black schizophrenic patients were institutionalized and categorized as defective when animal slurs were used to describe them/their behaviors, specifically linking their disability and identity to racism and animalization (122). Taylor (2017) recounts how as a child, she was excluded from joining other children in games and activities because she walked "like a monkey" - one of many instances in which she is compared to an animal because of her disability (104). This is similar to how individuals with vitiligo are not only compared to animals but also excluded from activities.

The practice of stigmatization is an important factor and central to the treatment and labeling of individuals with disabilities and conditions. These labels are used to make them outcasts. One such comparison is of Taylor (2017) and Clare (2017) who were both compared to monkeys because of their disability. In my research, I found that these labels occur because of stigma, and with vitiligo, individuals are subjected to slurs and animalization. But there is another problem that arises with stigmatization and labeling, and that is whether vitiligo should be considered a disability or condition and the treatment one should experience. The categorization of vitiligo as a disability or condition by medical science 
poses problems of ambiguity, since dermatologists and individuals with vitiligo do not agree on how to categorize it themselves. Research done by Murray (1996) under the Global Burden of Disease (GBD) tried to find classifications to qualify vitiligo as a disability. Murray's (1996) classifications measured disability weights for nonfatal health states on a scale from 0 (no disability - perfect health) to 1 (complete disability-death) (as cited in Carmichael 2016, 207). The finding acknowledges vitiligo on the face causes social exclusion from group interactions due to one not being physically attractive because of their vitiligo. But other than that, vitiligo is categorized as nothing serious, ranging from 0.00 to 0.02 (Carmichael 2016, 207). Murray (1996) does take into account that in certain cultures vitiligo can be mistaken for leprosy, which causes it to be considered contagious by the culture and hence, prone to be categorized as a disease (as cited in Fewtrel \& Bartram 2001, 47).

Individuals with vitiligo themselves debate with each other on whether to call vitiligo a condition or a disease. During the conference, there was a panel where participants spoke about cultivating relationships, but this topic gave way to the debate on disease or condition. Alice, a white woman who had developed vitiligo at a young age, spoke of how she wants vitiligo to be called a disease, so it can gain recognition, as other conditions such as eczema. Alice's point of view was met with agreement from a few audience members, who shared that calling vitiligo a condition makes it sound like a non-issue and medical practitioners would, therefore, pay it no mind. This perspective was also met with disagreements. One audience member, a man in his 30s, compared vitiligo as a disease to a car. He stated "Calling vitiligo a disease means there something very wrong with the person. To me, it's like a car's parts - if there is something wrong with the car, it needs to be replaced or else it doesn't perform the way it should. By calling vitiligo a disease makes it sound as if we have a disease comparable to AIDS or cancer." The words of both Alice and the man at the conference show that individuals with vitiligo struggle to categorize vitiligo.
Struggling to identify vitiligo as a condition or disease suggests real-world consequences. Calling vitiligo a disease prompts medical practitioners to pay attention to it, whereas calling it a condition leads to it being considered an 'orphan condition', a rare condition that is under researched because it does not create a market large enough to warrant attention and the development of treatments. Categorizing vitiligo as a disease inclines doctors to work on a cure which would lead to understanding it as a serious medical issue. However, identifying vitiligo as a disease would lead people with it to experience more stigma, prompting animalization and ostracization. On online platforms such as Reddit, some users of the site held discussions where they referred to vitiligo as a disease, specifically calling it Michael Jackson disease. Describing vitiligo as a disease and identifying it with a celebrity gives it validation and a face that the general public can notice and recognize. This, of course, brings awareness and illustrates that it is not a nonissue; rather vitiligo is a serious condition, leading to hardships such as loss of master status. It can affect anyone, even well-known celebrities. On Instagram, I saw posts mainly consisting of how to deal with vitiligo and individuals sharing thoughts and feelings concerning the condition. These posts prompt discussions that have a similar format as support sites. To answer the question concerning if vitiligo is a disease or a condition mainly reflects on whom one asks and how the individual chooses to identify it as. Yet, we need to bear in mind that either category has realworld consequences associated with it; vitiligo as a disease means it is contagious and lifethreatening, but instead allows more recognition in the medical community, whereas, referring to vitiligo as a condition would lead it to be considered a non-threatening skin condition and lead to less recognition in the medical community.

The perception that a cure results in devaluing the current self makes people with disabilities believe that in their current state they do not fit into a distinct model of normal; thus, society would not accept them (Clare 2017, 55). In the documentary Fixed: The Science/Fiction of Human Enhancement (2013), participants with disabilities shared that they 
would rather not have a cure for their disability but have solutions to help them get around the barriers those disabilities create in their lives, such as stairs by installing wheelchair ramps or developing automatic wheelchairs with longer battery life, thus, making it easier for individuals with disabilities to be accepted within the larger culture. Similarly, I asked my participants if a cure for vitiligo was developed would they take it, to which many replied they would not. When I inquired as to why not, they exclaimed their vitiligo is a part of them and if it was to disappear they would lose an important part of their identity. According to Richard, "This condition has given me life experiences I would not have had. I got to know people for who they really are due to this condition, and I'm grateful for that." Therefore, having vitiligo has made individuals with it understand people better because they know if a person around them genuinely appreciate them and does not feel embarrassed by their condition. As noted by Erika, "This is the only skin I know, and it took a lot to finally accept it and I would not trade that for the world." This shows how, for many, vitiligo is an experience that has given them hardships, but they are able to overcome those hardships and accept themselves. In some cases, people are happy that they have vitiligo and they experience the world differently and they would not change that because it makes them who they are.

\section{Findings: Vitiligo Discourses}

Using participation observation, social media, and informational interviews, I uncovered three discourses or themes that kept recurring. These discourses were: 1) Feeling Outcast, 2) Vitiligo as beautiful, and 3) Solidarity.

\section{Feeling Outcast}

People with vitiligo are often compared to animals and called names; subsequently, individuals are asked if the condition is contagious. Individuals with vitiligo are bullied, stigmatized, and experience verbal slights and overall, they are often ignored. At the conference, Thomas, one of the presenters, stated how individuals with vitiligo are compared to cows or Dalmatians, and then alluded to Erika, a fellow presenter, who had stated she was called an Appaloosa (Spotted Horse) by a close friend. These common animal comparisons prompted the audience to call out or talk among themselves about the animalization they endured.

During my interviews, a few participants spoke about feeling like outcasts, either by not being invited to events or as a result of friends pretending not to know them outside of school or the workplace. One of my interviewees, Richard, who is in his late 30s, reminisced about his friend not inviting him to his birthday party when he was in elementary school; When Richard inquired as to why he was not invited, the friend answered, "My mom thinks that what you have will spread to the others." Through this experience, Richard fully understood he was different from others. Other interviewees shared similar experiences. Another presenter at the conference, Jasmine, shared two instances in which she experienced discrimination while on a public bus. The first was recounted in the introduction. In the second, when Jasmine got on a bus, a passenger seated across from her kept staring at her, leading her to switch her seat, which led more passengers to stare. After Jasmine moved to the back of the bus, she noticed the man still staring at her. She stated, "I felt embarrassed being stared at. It's not as if I'm not used to these stares; it's just at this time in our history. I think people should be used to seeing vitiligo or any condition. By being stared at, it made me feel like I wasn't allowed on the bus."

This notion of staring or often feeling uncomfortable or even fearful around people with disabilities, as if the disabling condition is contagious, results in individuals with it being othered. As presented by Murphy (1987), “...all too many non-disabled people view people with disabilities as a "fearsome possibility'"' (as cited in Beger 2013, 8); therefore, the disabled person becomes the "other - a living symbol of failure or frailty...a counterpoint to normality; a figure whose humanity is questioned" (Beger $2013,8)$. This display of fear of the impairment's contagiousness could be seen in Jasmine's fellow passengers, where they questioned if they too could develop vitiligo and if she was "normal." At the conference, when the topic of feelings of isolation came up, a young lady, Nita, noticed that she was "apologizing" for her condition because of the way many people reacted to it. But, as she put it, vitiligo is a part of her; thus, she should not apologize for it. 
Participants shared that they feel ignored by people they knew. Richard mentioned that, as a teenager, when he saw his friends on the streets and approached them they pretended they did not know him. With this, Richard went on to share another experience of rejection from a girl he liked because of his condition. He recounted asking her to the school dance, to which she replied she would be embarrassed to go with him because of his vitiligo. Being rejected based on their appearance was an experience shared by other participants; Jasmine shared that a man she did not know stated, "It must be hard to look at yourself in the mirror." While Alice spoke about growing up with vitiligo, she was considered different by others in her community, even though she is white and her vitiligo is barely noticeable. Yet, her peers would point out her condition and treated her as an outsider. Alice's case is similar to Berger's (2013) concept of individuals whose bodies are different from "a society's conception of a 'normal' or acceptable body," and how, even when this causes "little or no functional or physical difficulty for the person who has them, [it] constitutes [as] major social [disability]" (8). In the case of facial scars or disfigurements, such a disability of appearance is only constructed by stigma and cultural meanings (Berger 2013, 8). Melody, on the other hand, expressed that she has not faced any negative acknowledgment from the community around her, but she does feel her family tends to see the condition negatively due to Melody being the only one in her family to have vitiligo. It is to be noted, other participants shared that they have family members who have vitiligo and face some or no negative reactions from family. Melody explains there are times at family gatherings when she leaves the room to use the bathroom and stares at herself in the mirror, wondering how her sisters and parents perceive her with vitiligo. Furthermore, Melody shared that during these times she contemplates how her life would be different if she did not have this condition. These findings indicate that each of my participants had faced/dealt with negative emotions due to their vitiligo. Individuals reported feeling isolated, stigmatized, animalized, and overall, feeling they were excluded from the community.

\section{Vitiligo as Beautiful}

Many participants at the conference perceived their vitiligo as nothing, passing it off as "just a skin condition" that did not put their lives at risk - most considered vitiligo as unique and beautiful. However, understanding vitiligo in such manner did not come easy. Participants shared negative experiences such as animalization or ostracization. Some attendees also indicated that they came to the event to learn about medical advancements, but at the end of the event, they left learning their condition grants them a uniqueness. A compilation of pictures of individuals with vitiligo, presented by Jasmine, displayed the various faces of the condition and exhibited the beauty vitiligo has, categorizing the condition as art on the body. In the collection, one picture captured my attention, a black and white picture which depicted a young AfricanAmerican girl who looked to be in her late teens in a white dress and her vitiligo was covering her eyes, making it appear as she was wearing a mask. This picture revealed to me that vitiligo was more than just a skin condition; it was art on the body and represented people's identities, which I did not consider until this moment.

However, before individuals are able to accept themselves, they must overcome barriers associated with changes in skin color. According to Erika, "It's hard to love your spots, and I'm not sure if I will ever love or like them, but I need to own them and live my life and make the choice to be happy with my vitiligo." What Erika mentioned is what many people struggle with when vitiligo develops or spreads. Many of the participants questioned why this was happening to them, if it was a transformation for the good, or if they would be "normal" again. Jasmine mentioned her friends defined her vitiligo as art on her body, which pushed her to consider her vitiligo as a transformation into a new her. However, two of my interviewees, Melody and Rachel, expressed periodically missing their old face. Melody said, "I used to be impacted by this condition in the morning and missed my former face, but there is nothing I can do about it now, so I live with what I got." Rachel spoke of how her pale white skin becomes tan during the summer causing her vitiligo to be noticeable, leading her to not 
recognize her own hands. But once individuals overcome the barrier of stigma and ostracization, they accept their vitiligo.

Perceiving the condition as art is empowering. Dawn, an interviewee, and model for the vitiligo community reminisced about when she stopped covering up her face. Dawn shared that one day, someone pointed out she has a "heart on her face" and this statement took her by surprise, stating she lived with this face for years, covering it up with makeup. This example exhibited how it was hard for her to come to terms with her vitiligo, but after this statement was uttered, it resonated with her coming to terms with her condition and prompted her to stop covering up her face. Upon sharing this information, Dawn showed me pictures of herself with makeup and mentioned she was not smiling and not happy because she had not accepted herself. This was evident in her pictures; Dawn appeared stonefaced compared to pictures without makeup, displaying her vitiligo with pride. While showing me these pictures, she stated vitiligo gave her a uniqueness that very few individuals possess, and it prompted her to finally embrace her condition. Dawn further shared her original skin color is coming back on her eyelids. She noted when washing her face, she noticed brown specks on her eyelids, initially assessing it was dirt and tried rubbing it off, but when it did not come off, she realized it was her original skin color. Dawn mentioned she does not like how her skin color is changing back to its original color since it took her a long time to come to terms with her vitiligo and finally accept herself and the condition. If she gets her original skin color back, she feels she will lose herself again. Through Dawn's experience, it is evident that even people with vitiligo do not see the condition as a uniqueness at first, but as they accept it, it becomes a part of their identity.

During Thomas's presentation, women spoke out about feeling beautiful because of their condition and noticed more people approaching them, especially men. Nita chimed in about her partner finding her confidence in her vitiligo attractive. Thomas too shared an instance of a young woman adorned in tattoos approaching him and tracing her finger to his cheek, notably on his vitiligo, prompting her to ask, "Where did you get it done from?" to which Thomas replied, "It was free." This anecdote displayed how the condition can be viewed as beautiful, even though the young lady confused Thomas's vitiligo for a tattoo. This raises the question of whether the young lady would have still considered vitiligo beautiful upon learning it is a skin condition. Perhaps she might have still perceived the condition as beautiful, but the label of 'condition' may also have changed her perspective.

Although most of the individuals I interviewed maintained that they felt confident with their vitiligo, many of them admitted that negative feedback from people could wear on them. The concept of the gaze can be used to understand how the public can stigmatize and ostracize individuals with vitiligo. As presented in Garland-Thomson's (2005) research on the historical context of the word "freak", the idea of the gaze provides insight as to how individuals with abnormalities can be socially 'othered' by people stigmatizing, ostracizing, and animalizing them. During her research, Garland-Thomson (2005) discovered in the 1960s a freak show called the Spotted Boys, which provides insight as to how individuals with vitiligo were portrayed as exhibits and subjected to the gaze of spectators (1-2). These individuals were considered spectacles to mesmerize and evoke curiosity or fear of the unknown to the public. The gaze represented by the spectators is a form of oppression that carries complex cultural and historical meanings which are often found in current discussions of the "male gaze" (GarlandThomson 2005, 2). At the conference, women with vitiligo shared experiences where they were subjects to the male gaze due to their condition and their gender. Many women were victims of slurs utilizing animal references designed to shame them as both women and a person with vitiligo. An example would be comparing a woman to a cow because of her vitiligo also references her body size. Moreover, Garland-Thomson's (2005) research introduces the concept of the medical gaze designed to pathologize the bodies of individuals with vitiligo. The medical gaze observes the condition as if to determine a diagnosis rather than distinguishing it as revolting or transforming, as considered by the general 
public (Garland-Thomson 2005, 3). Through this perspective, individuals with vitiligo are characterized as sick, which can evoke fear of contagion. The male gaze is utilized on individuals with vitiligo, where they might be considered less attractive because they have white patches on their skin. From my research, I found that women had endured this challenge of being othered and learned to accept their vitiligo as a part of themselves (further discussed below).

These "freak shows" allowed spectators to become spectacles as described by Berger (2013). He illustrates that people with disabilities and impairments who were exhibited at such shows were allowed to stare back at those non-disabled individuals who stared at them (Berger 2013, 3). This turned society's gaze back onto itself to show nondisabled individuals that their "normal" is not "normal" in the eyes of individuals with disabilities. As illustrated by Davis (1997), nondisabled people "see themselves as living in a mirage of being normal" (As cited in Berger 2013 , 3). What Davis (1997) is inferring is that non-disabled people live out their lives thinking that they would not be affected by disability that they are "normal" and will be normal until the end. But people without disabilities need to understand disability as a social phenomenon that helps us all to see how interconnected human beings are and how very much it diminishes us to assume that any life is without value.

Individuals with vitiligo share that accepting and loving one's vitiligo is a journey that is filled with heartache - that starts with learning there is no cure, being stigmatized and ostracized by friends, family, and community, and feeling alone. However, as they live their day to day life with vitiligo, overcoming barriers, people with vitiligo start to see the condition as a part of themselves. Some feel empowered by the condition and calling it art on their skin, and making it an integral part of their identity.

\section{Solidarity}

At the event, I noticed attendees running up to other attendees who entered the hall and pulling those individuals into a hug. Initially, I thought this was done because the attendees were friends or acquaintances who had not seen each other in a while, or had met at previous conferences. But as I continued to observe, the individuals pulled away to introduced themselves and proceeded to ask the others' names, where they were from, and communicate how great it was for them to be a part of the event. This indicated individuals with vitiligo have social solidarity, as mentioned by Jasmine. Social solidarity at the conference illustrated that two people with vitiligo, who were from different walks of life, were able to have a mutual understanding of what the other person might have gone through: the trials and tribulations of having vitiligo.

The concept of social solidarity, or an unspoken bond, was evident throughout my research, as I observed how individuals spoke or joked about their condition. I recall a moment at the conference where I stood with Dawn and her close friend Michelle whom she met through these annual conferences; they were talking about their vitiligo, specifically on how it has spread. Dawn indicated her vitiligo has not spread but she has noticed brown specks (her original skin) coming back, to which Michelle replied that her vitiligo had not changed and jokingly compared the white streaks in her hair caused by vitiligo to a skunk's tail; as a result, Michelle covers up the top of her head with a bandana. As Dawn and Michelle's interaction took place, I saw how both ladies were open to talking about their vitiligo - where they joked and shared concerns they had regarding the condition. This is a display of social solidarity, where two individuals formed solidarity around a condition by sharing concerns that they knew only a person with vitiligo could understand. Even Michelle's comparison of her hair having white streaks like a skunk's tail illustrated her creating solidarity with Dawn by referring to the animalization people with vitiligo are subjected to - being called a cow, Dalmatian, or appaloosa.

Social media accounts on platforms such as Instagram and Facebook are dedicated to vitiligo. This assists in connecting individuals to others with the condition, forming communities around common experiences, and developing support outlets. The Instagram account called Vitiligo-Beauties hosts pictures submitted by 
people with vitiligo to the user of the account to showcase the pride people have in their condition. Through this account, there is immense vitiligo pride on display, and in the comment section, users of the site find support and share stories of their experiences with the condition. People with vitiligo not only display pride in their condition but also find common ground and formulate social solidarity. This practice of pride is similar to LGBTQ and Disability pride, where individuals are claiming the word vitiligo as their identity, not as an unfamiliar entity on their skin. In comparison to LGBTQ pride, the word "queer" is used within the group to create solidarity with one another, whereas Disability pride utilizes "Crip" in the same sense (Taylor 2017, 132). These words were once utilized by the general public to "other" those with disabilities or nonheteronormative sexual orientations. But now individuals from these communities have claimed these words to highlight their sexuality or disability as an affirmative identity and show the common cause of a particular political constituency (Berger 2013, 5).

Solidarity and acceptance of the condition is not only done through reclaiming words such as vitiligo to formulate one's identity. While on the Instagram page of Vitiligo-Beauties, I found two images that stood out to me. The first one depicted the back of a hand with vitiligo and in the middle of the hand is a tattoo of a cow. This image portrays how the individual associated their condition with the animal that is famous for its white patches. The individual displayed and embraced their vitiligo and the connotation of being called a cow. The comments on the image read it is an inspirational tattoo, in the sense that cows are used in animalizing people with vitiligo; thus, only individuals with vitiligo understand its significance. Some commenters also shared their wish to acquire a similar tattoo to display their vitiligo pride. In the second image, there was another hand, but the vitiligo was outlined by markers of various colors. This image caught my attention since Erika shared an experience where her friends colored in her vitiligo with gel-pens, and she did not like how the colors defined her spots. But in the comments section, many users shared that they color in their spots with only one color, while others shared an interest in wanting to color in their spots with multiple hues, and some found the image pleasing. With this noted there were times individuals shared stories and requested that each other exchange email addresses to be better acquainted. Displaying how individuals form communities utilizing social media, how they connect over pictures, share their experiences, and reclaim the word vitiligo and the animals used to other them to formulate an identity makes people with the condition feel empowered.

Jasmine and Erika shared during the conference that they have established platforms for individuals with vitiligo. Jasmine mentioned she connects with people through Instagram, where she posts pictures of her vitiligo and of people with the condition she meets in her daily life. By building support from social media, people with vitiligo assist one another to come to terms with their condition and see the beauty and uniqueness their spots have to offer. Jasmine has organized a support group called "Tough Skin," dedicated to not only help individuals with vitiligo but also people with cerebral palsy or depression and various other conditions, providing them with a support group where they can form communities around common experiences. Similarly, Erika is the co-founder of "Living-Dapple," a blog where individuals document, share and support each other on their journey with vitiligo. The website hosts tips to build confidence, accept one's vitiligo, and overall, to advocate for the condition. Living-Dapple also has an Instagram account dedicated to inspirational quotes, artsy/artistic and aesthetic pictures, and dialogue on individuals' experiences with the condition (from asking questions about how one feels when finding a new spot to dating and relationships). These women, like many others, are working to build awareness for vitiligo by connecting people with the condition to others like themselves, to reduce the impact of stigma and isolation and have a community where they belong too.

In addition to online support, support organizations such as VIT-Friends and V-Strong establish a sense of family, where individuals bond over their condition and share their experiences face to face. These sorts of organizations are located across the country. 
VIT-Friends is located in NYC and wishes to connect all vitiligo chapters under its umbrella. $\mathrm{V}$-Strong is based in Michigan and has recently partnered with VIT-Friends, becoming one of its chapters, and in addition hosted the 2017 conference. Through these forms of connection, individuals with vitiligo have a place where people understand their struggles, connect on common experiences, and get help with accepting the condition. This harkens back to Erika's sentiment about the necessity of coming to terms with vitiligo. It is hard for people with vitiligo to love their spots but in the end, identify with them allowing them to be a happier person.

Vitiligo has provided people not only with negative experiences and emotions but also with positive ones. My informants alluded that they experienced loss of self when they first developed vitiligo. But as they lived with their vitiligo, they learned to accept it and own it as a part of their identity. Through this condition, they were able to create solidarity with others with similar experiences regarding stigma, ostracization, and animalization.

\section{Analysis: Beyond the Words}

While participants in my study referred to vitiligo as "nothing," the emotional and personal stories I heard at the conference and through interviews suggested that the condition was anything but. Vitiligo is extremely consequential in terms of individuals developing depression or feelings of isolation. Participants walked a fine line between distress and pride, which was evident in their stories. Jasmine was a model when she developed vitiligo; she felt alone, depressed, and she constantly asked why she had developed the condition. Erika shared similar experiences, where she hated the way she looked. She thus held onto hope that one day there would be a treatment; she would be "normal" and would not have to hide her spots. But as time passed, she realized there was no cure and that she would have to live with these spots for the rest of her life, leading her to cry for long periods of time.

No two people see their vitiligo in the same way. For some individuals, vitiligo is beneficial because the general public can see beyond it and not outcast them for it; for others, it is a major problem because they experienced stigma and isolation, which they wouldn't otherwise. Aside from feeling isolated, women at the conference also expressed that their vitiligo was alluring. A few women during a panel stated they noticed attracting the attention of men who would show romantic interest in them. As Nita shared, her boyfriend was interested in developing a relationship with her because she carried herself with confidence. By this, he meant the confidence Nita had in her vitiligo because she owned the condition as a part of herself. This touched her and assisted her in fostering their relationship. Similarly, Melody also shared that she developed vitiligo before she started dating her now fiancé, and he said her vitiligo makes her memorable. By contrast, when I was chatting with Dawn and Michelle, they spoke about the negatives of vitiligo and how hard it is to hide it when finding a partner or interviewing for a job. They mentioned covering vitiligo with makeup was not useful since the makeup would not last all day, especially when you have vitiligo on your hands. In general, the informants said when they put makeup on their hands, it got washed off and then people stared at their hands. While some women feel vitiligo is as an asset, my male informants' spoke of how it is hard to find a partner. They spoke about the hardship of hiding their vitiligo with makeup for fear of facing backlash from society. Richard started having difficulty formulating relationships because he did not want to inconvenience his partner with the stares he received over his vitiligo. As illustrated by my informants, vitiligo is experienced differently by everyone. For some it is alluring or an asset while for others it is an inconvenience.

The concept of black individuals with vitiligo being affected more severely by stigma compared to their white counterparts was explored in Porter and Beuf's (1991) research, and my study reinforces this finding. When I was at the conference and approached potential informants, I found that white or lightskinned attendees often said: "I am not sure how my experience would be beneficial to the study." They further stated that they were not affected with too much stigma or did not have the overwhelming experience of losing their racial identity, as people of color encounter. 
Individuals who identified as white or fair skinned mentioned their spots were only prominent during the summer when they were tan, and this is when they would foster stares. They also shared that most times the public could not notice their vitiligo, due to it not being prominent, and when they did notice, they considered it as light discoloration. However, my non-white informants mentioned facing the issue of losing their master status and oftentimes feeling they no longer belong or are a part of their racial group. People of color are often faced with questions regarding what vitiligo is, how they developed it, and subjected to personal questions on where on their body these spots are prevalent, in comparison to their white counterparts. It is important to note that there is more stigma around vitiligo for people of color because the condition is more prominent on their skin in comparison to fairskinned individuals. Therefore, many people of color are subjected to ostracization from their racial groups, losing their racial identity and a sense of who they are.

Lastly, vitiligo is not inherently an issue but becomes one through social stigma resulting from depigmentation of the skin that raises concerns about the unknown from the public. Individuals across the country and sometimes across the world gather to hold a yearly conference around it. They have established support groups and sites dedicated to documenting individual's journeys and aiding others in coming to terms with their vitiligo. The idea of how vitiligo is a non-issue comes from calling it "nothing," which was discussed at length in my research and in the research of Berger (2013) in regard to disability. But as I have argued, vitiligo is anything but a non-issue. Based on the research summarized in this paper and the work of other scholars, it is proven that vitiligo is a series of multidimensional issues that not only affect the physical self, but the emotional and psychological self too. Vitiligo as a condition is very much an issue to the people who have it and it plays a critical role in their lives. These individuals experience stigmatization and ostracization, and from these occurrences they build on how they identify with the condition, thus owning it. While vitiligo is considered beautiful by many who have it, it is a complex condition that leads to stigma and exclusion.

\section{Conclusion}

Though they might face negative reactions due to their condition, my participants indicated the mentality surrounding vitiligo is changing. In my research, I noticed a recurring theme: "It is a skin condition, which is hard to love at first, but it makes one memorable and beautiful." This statement was uttered throughout the conference; participants felt proud of their condition. When I was interviewing Jasmine, she recounted to a time when she was younger and saw a woman of East Asian origin who had vitiligo on the side of her face and, because of the vitiligo, some of her hair had turned white, as Jasmine described. She went on to state she found this unknown lady beautiful and, fifteen years later, she developed vitiligo herself. Jasmine shared that the general public's perception of vitiligo is changing due to fashion icon Winnie Harlow, who has brought light to the matter. Dawn and Michelle mentioned that years ago, they never thought people like them, with vitiligo, would have the chance to be models. Winnie Harlow gracing the covers of magazines and billboards has aided in changing beauty norms, despite the preconception that models should conform to a certain body image.

In fact, models with vitiligo are now the face of diversity. Because they have a range of skin tones within just one body, they can be utilized to show the face of vitiligo, as in the recent Cover Girl ad. Rather than choosing one shade for her face, and covering up the lighter skin patches that mark the vitiligo, new Cover Girl, Amy Deanna chooses two different foundations, claiming, "Why try to blend in when you can choose how to stand out?"1 This ad campaign highlights that cultural norms are changing and reinforces my claim that people with vitiligo learn to love their difference and feel valued for it. Further, ads like these make it possible for other individuals with vitiligo to feel empowered and understand their condition does not define them but is a part of them.

Consequently, this research is timely in that it resonates with our current cultural climate, due to the key issues regarding race and stigma, as well as, gender, identity, and acceptance or opportunities that are 
experienced by individuals with vitiligo. We see that more and more individuals are changing the public's perception of vitiligo from a stigmatizing condition to a condition that is unique and celebrated. We see this occurring especially in the entertainment industry, where individuals such as Winnie Harlow from America's Next Top Model, Amy Deanna as the Covergirl model of 2018, and Zazie Beetz as Domino from Deadpool 2, are overcoming stigma and changing our perception of the condition and creating an identity around vitiligo. Through this, individuals are empowered and bring awareness to the condition.

With only 1 percent of the world's population affected by vitiligo, it may be tempting to overlook it as a social or medical issue. However, my participants' stories showcased how individuals are stigmatized and ostracized by their communities. While some of the participants felt that society negatively impacted their perceptions of themselves and their vitiligo, those negative feelings were not freely expressed by participants, who only spoke of them when they were prompted to elaborate on their experiences. Through vitiligo, individuals overcome these hardships and come to terms with the condition, viewing it as a fragment of their being. Many participants shared experiences and posted pictures on the condition online; thereby forming a community and assisting others in accepting the condition and becoming comfortable with it themselves. Ultimately, additional research is needed on the topic and its effects on transforming people's lives. 


\section{Acknowledgements}

I want to extend my special thanks of gratitude to my brother and my mother; the World Vitiligo Conference and its participants for sharing their experiences with me during the course of this research; and my professors Dr. Elaine Gerber and Dr. Maisa Taha who provided the expertise that greatly assisted the research. These people helped me to complete the writing of this article as well as the challenging research that lies behind it.

\section{Endnotes}

1. Similarly, a new commercial from CoverGirl for its \#IAmWhatIMakeUp truBlend Foundation features Amy Deanna, the first model with vitiligo that the company has used in their advertising. http://www.dailymail.co.uk/ femail/article-5511449/AmyDeanna-CoverGirls-modelvitiligo-talks-campaign.html 


\section{References}

Berger, Ronald J. 2013. Introducing Disability Studies. New York: New York University Press.

Brashear, Regan, dir. 2013. Fixed: The Science/Fiction of Human Enhancement. New York: New Day. DVD.

Campbell, Fiona A. Kumari. 2008. “Exploring internalized ableism using critical race theory, Disability \& Society." Journal of Disability \& Society 23(2): 151-162.

Carmichael, Gordon. 2016. Fundamentals of Demographic Analysis: Concepts, Measures and Methods. New York: Springer International.

Clare, Eli. 2017. Brilliant Imperfection: Grappling with Cure. Durham: Duke University Press.

Davis, Lennard. 2006. The Disability Studies Reader. New York: Taylor \& Francis.

Domino, Frank J, Baldor, Robert A., Grimes, Jill A., and Golding, Jeremy. 2014. The 5-Minute Clinical Consult Standard 2015. City: Lippincott Williams \& Wilkins.

Durkheim, Emile. 1997. The Division of Labour in Society. Translated by W. D. Halls. New York: Free Press.

Fewtrell, Lorna and Bartram, Jamie. 2001. Water Quality Guidelines, Standards and Health: Assessment of risk and risk management for water-related infectious disease. World Health Organization.

Garland-Thomson, Rosemarie. 2005. "Staring at the other." Disability Studies Quarterly25(4): 1-3.

Goffman, Erving. 1963. Stigma. New York, NY: Penguin.

Koo, John Y. M. and Lee, Chai Sue. 2003. Psychocutaneous Medicine. New York, Basel: Marcel Dekker.

Lundby, Knut. 2009. Mediatization: Concept, Changes, Consequences. New York: Peter Lang.

Oliver, Michael. 1996. “Understanding Disability: From Theory to Practice." The Journal of Sociology \& Social Welfare 23(3): 193195. 
Parens, Erik. 2006. Surgically Shaping Children: Technology, Ethics, and the Pursuit of Normality. Baltimore: John Hopkins University Press.

Parsad, Davinder, Dogra, Sunil, and Kanwar, Amrinder Jit. 2003. "Quality of life in patients with vitiligo." The Journal of Health and Quality of Life Outcomes 58(1): 1-3.

Porter, Judith R. and Beuf, Ann Hill. 1991.“Racial Variation in Reaction to Physical Stigma: A Study of Degree of Disturbance by Vitiligo Among Black and White Patients." The Journal of Health and Social Behavior 32(2): 192-204.

Reinecke, Leonard and Oliver, Mary B. 2016. The Routledge Handbook of Media Use and Well-Being. New York: Taylor \& Francis.

Sergeant, Philip. and Tagg, Caroline. 2014. The Language of Social Media: Identity and Community on the Internet. New York: Palgrave Macmillan.

Shah, R., Hunt, J., Webb, T.L., and Thompson, A.R. 2014. "Starting to develop self-help for social anxiety associated with vitiligo: using clinical significance to measure the potential effectiveness of enhanced psychological self-help." British Journal of Dermatology 171(2): 332-337.

Taylor, Susan. 2017. Beasts of Burden Animal and Disability Liberation. New York: New Press.

Titchkosky, Tanya and Michalko, Rod. 2009. Rethinking Normalcy: A Disability Studies Reader. Toronto: Canadian Scholars' Press. 